\title{
CONTRIBUIÇÃO DOS PONTOS DE ENTREGA VOLUNTÁRIA COMO INSTRUMENTO PARA O FORTALECIMENTO DA GESTÃO MUNICIPAL E PRÁTICAS DE COLETA SELETIVA: uma análise bibliométrica
}

Versão do autor aceita publicada online: $21 \mathrm{dez} .2021$

Publicado online: 06 jan. 2022

Como citar esse artigo - American Psychological Association (APA): Viña, F. S., Kipper, L. M., \& Moraes, J. A. R. (2022). Contribuição dos pontos de entrega voluntária como instrumento para o fortalecimento da gestão municipal e práticas de coleta seletiva: uma análise bibliométrica. Exacta. DOI: https://doi.org/10.5585/exactaep.2022.20732.

\section{Franciele Stoffel Viña}

https://orcid.org/0000-0002-2945-7528

Universidade de Santa Cruz do Sul

Estudante de Engenharia de Produção

\section{Liane Mahlmann Kipper}

http://orcid.org/0000-0002-4147-892X

Universidade de Santa Cruz do Sul

Bolsista produtividade CNPq. Professora titular da Universidade de Santa Cruz do Sul e Coordenadora do Mestrado em Sistemas e Processos Industriais de 2013 até 2015. Atua na graduação e na PGSS (PPGSI, PPGPSI e PPGTA). Nas áreas de gestão do conhecimento, inovação e criatividade, e em gerenciamento de processos e métodos e técnicas de pesquisá desenvolve ensino, pesquisa e extensão com foco em: melhoria e gestão de processos, sistemas enxutos e indústria 4.0: inovação, criatividade, design thinking, deisgn science research, desenvolvimento de produtos e tecnologias para otimização e melhoria de processos. Tem também experiência na área de Física e Física Experimental. De 1995 até 2009 atou junto a gestão universitária em especial nas áreas de pesquisa e pós-graduação (lato sensu e stricto sensu). Atua na extensão da área tecnológica e educacional e no TECNOUNISC coordena o Laboratório Interativo de Criatividade (LIC). ResearcherID: F-8937-2013 Scopus Author ID: 12143252800

\section{Jorge André Ribas Moraes}

https://orcid.org/0000-0002-9505-8883

Universidade de Santa Cruz do Sul

Possui graduação em Engenharia Mecânica pela Universidade Federal de Santa Maria (1988), Mestrado em Engenharia de Prođução pela Universidade Federal de Santa Maria (1998) e Doutorado em Engenharia de Produção pela Universidade Federal de Santa Catarina (2005). Atualmente exerce a função de chefe do departamento de Engenharia Arquitetura e Computação da Universidade de Santa Cruz do Sul - UNISC e é professor do Programa de Mestrado e Doutorado em Tecnologia Ambiental PPGTA da UNISC. Professor tempo Integral da Universidade de Santa Cruz do Sul. - UNISC. Tem experiência na área de Engenharia Sanitária, com ênfase em Gestão Ambiental, atuando principalmente nos seguintes temas: produção mais Limpa; gestão ambiental; gestão de riscos, qualidade e produtividade; segurança do trabalho e ergonomia; reciclagem de materiais e melhoria de processos.

Resumo: Os Resíduos Sólidos Urbanos (RSU) necessitam de uma gestão adequada, pois envolvem o cuidado com o meio ambiente e a saúde da população. O objetivo da pesquisa é mapear a evolução do conceito de Ponto de Entrega Voluntária (PEV) e suas relações com o 
processo de reciclagem dos RSU; avaliar como a Política Nacional de Resíduos Sólidos (PNRS) contribuiu para a disseminação das Cooperativas de Materiais Recicláveis e como a gestão municipal pode utilizar os PEVs como instrumentos para o fortalecimento das práticas da coleta seletiva no Brasil. A gestão municipal, utilizando os 16 indicadores selecionados, poderá estruturar PEVs, atendendo a PNRS, e qualificar a gestão dos RSU. Essa ação contribuirá também para melhorar a qualidade de vida dos catadores de materiais recicláveis, elevará a educação ambiental de toda a população afetada e reduzirá desperdícios de materiais que podem ser reintroduzidos na cadeia de produção e consumo.

Palavras-chave: Pontos de entrega voluntária. Gestão Municipal. Reciclagem. Coleta Seletiva. Cooperativas.

\title{
CONTRIBUTION OF VOLUNTARY DELIVERY POINTS AS AN INSTRUMENT FOR STRENGTHENING MUNICIPAL MANAGEMENT AND SELECTIVE COLLECTION PRACTICES: a bibliometric analysis
}

\begin{abstract}
Urban Solid Waste (USW) needs proper management as it involves care for the environment and the health of the population. The objective of the research is to map the evolution of the concept Voluntary Delivery Point (VDP) and its relationship with the USW recycling process; evaluate how the National Solid Waste Policy contributed to the dissemination of Recyclable Materials Cooperatives and how municipal management can use VDPs as instruments to strengthen selective collection practices in Brazil. The municipal management, using the 16 selected indicators, will be able to structure VDPs, meeting the National Solid Waste Policy, and qualify the management of the USW. This action will contribute to improving the quality of life of recyclable material collectors, will raise environmental education for the entire affected population, and will reduce wasted materials that can be reintroduced in the production and consumption chain.
\end{abstract}

Keywords: Voluntary delivery points. Municipal Management. Recycling. Selective collect. Cooperatives.

\section{Introdução}

Do lixo ao resíduo há um grande caminho a ser percorrido. Lixo é entendido como qualquer material sem valor ou utilidade, conceito que traz a percepção de inutilidade dos materiais após seu uso. Já a palavra resíduo se origina do substantivo masculino resto ou aquilo que resta. De acordo com Política Nacional de Resíduos Sólidos (PNRS), lei 12305/2010, resíduos são definidos como sendo todo material, substância ou objeto descartado resultante 
de atividades humanas em sociedade, ou seja, é considerado um bem, o que revela a existência de valor (Maiello, Britto \& Valle, 2018). O lixo urbano no Brasil, até 1780, era pouco diversificado, sendo formado basicamente por restos de alimentos (Batista, Oliveira \& Oliveira, 2016). Após essa data, o lixo começou a aumentar sua diversificação e composição gravimétrica, passando a ser mais do que somente restos provenientes da alimentação humana. Os Resíduos Sólidos Urbanos (RSU), no Brasil, começaram a ter gestão e tratamento a partir de 1980, porém foi um começo pouco desenvolvido quando comparado com outros países (Soares, Barbosa, Vieira \& Silva, 2020). Havia uma grande quantidade de lixo, não de resíduos, e pouco investimento, o que causou uma série de problemas na gestão municipal dós resíduos sólidos (Soares et al., 2020).

Nos dias atuais, os resíduos sólidos precisam ter uma gestão adequada, o que é visto como um desafio no âmbito da gestão ambiental, pois é um tema muito complexo e que envolve o cuidado com o meio ambiente e a saúde da população (Santiago, 2016). No Brasil ainda há uma falta de preocupação com a gestão dos RSU, sendo isto, uma questão histórica, e por isso, deve-se ter uma atenção especial no assunto (Soares et al., 2020). Santiago (2016) realizou uma comparação entre Brasil, Estados Unidos, Japão, União Europeia, América Latina e Caribe, verificando que o Brasil está quatro décadas atrás dos países mais desenvolvidos (Figura 1).

\begin{tabular}{|c|c|c|c|c|}
\hline & $\begin{array}{c}\text { Início do } \\
\text { Desenvolvimento } \\
\text { das Políticas }\end{array}$ & $\begin{array}{l}\text { Postura na } \\
\text { Gestão de } \\
\text { Resíduos } \\
\text { Sólidos }\end{array}$ & $\begin{array}{c}\text { Centralização do } \\
\text { Gerenciamento }\end{array}$ & $\begin{array}{l}\text { Principais } \\
\text { Desafios }\end{array}$ \\
\hline Estados Unidos & Década de 1970 & Proativa & $\begin{array}{l}\text { Sistema regional, } \\
\text { autonomia dos } \\
\text { estados }\end{array}$ & $\begin{array}{l}\text { Gestão de } \\
\text { resíduos } \\
\text { perigosos }\end{array}$ \\
\hline União Europeia & Década de 1970 & Proativa & $\begin{array}{l}\text { Descentralizado, } \\
\text { autonomia dos } \\
\text { governos locais }\end{array}$ & $\begin{array}{l}\text { Redução da } \\
\text { geração }\end{array}$ \\
\hline Japão & Década de 1970 & Proativa & $\begin{array}{l}\text { Descentralizado, } \\
\text { autonomia das } \\
\text { municipalidades }\end{array}$ & $\begin{array}{l}\text { Redução da } \\
\text { geração }\end{array}$ \\
\hline $\begin{array}{c}\text { América Latina } \\
\text { e Caribe }\end{array}$ & Após 2000 & Reativa & $\begin{array}{c}\text { Tendência de } \\
\text { descentralização }\end{array}$ & $\begin{array}{l}\text { Elaboração e } \\
\text { aplicação de } \\
\text { políticas } \\
\text { públicas }\end{array}$ \\
\hline Brasil & Em 2010 & Reativa & $\begin{array}{c}\text { Descentralizado, } \\
\text { autonomia dos } \\
\text { governos locais }\end{array}$ & $\begin{array}{l}\text { Aplicação da } \\
\text { política pública }\end{array}$ \\
\hline
\end{tabular}

Figura 1 - Comparação das estratégias de gestão de resíduos sólidos em diferentes regiões 
Fonte: Adaptado de Santiago (2016).

Analisando a figura 1, pode-se perceber que no Brasil, América latina e Caribe o principal desafio é a aplicação da política pública. Já nos países desenvolvidos, o foco é a redução da geração de resíduos, ou seja, redução na fonte poluidora. Isto revela a necessidade de estudos que buscam apresentar a contribuição de mecanismos, como Pontos de Entrega Voluntária (PEV) e ações colaborativas com as associações de catadores para mudar a gestão de resíduos no Brasil, América latina e Caribe. Há título de comprovação desta necessidade, os plásticos, no Brasil, são o segundo material mais recolhido por cooperativas e catadores, sendo necessário um plano de coleta adequado para que os materiais possam retornar para o processo de produção através da reciclagem ou reutilização (Kipper, Mahlmann \& Rodríguez, 2009; Associação Brasileira das Empresas de Limpeza Pública e resíduos especiaisABRELPE, 2020). Os autores ainda dizem que não existe somente uma definição para o ato de reciclar, mas ele pode ser considerado a ação de coletar, reprocessar, comercializar e utilizar materiais que seriam considerados lixo.

A reciclagem, como norteadora de uma educação ambiental inclusiva e participativa da sociedade, deve ter um papel fundamental na disseminação da coleta seletiva em todos os municípios do Brasil. Mesmo sabendo que ainda há muito o que ser feito em termos de gestão dos resíduos sólidos urbanos pelos agentes públicos, percebe-se um aumento em programas de coleta seletiva, pois segundo Batista et al. (2016) de 1989 a 2008 ocorreu um aumento de $17 \%$ no número de programas de reciclagem.

Algumas pesquisas indicam que, no Brasil, para 1 tonelada de lixo domiciliar, há até duas toneladas de resíduos de construção e demolição civil, sendo extremamente necessário o tratamento adequado destes, pois são resíduos que podem causar problemas ambientais e sociais (Fagury \& Grande, 2007). A indústria de construção é uma das que mais gera resíduos sólidos, considerando toda sua cadeia produtiva, sendo assim necessária a atuação das usinas de reciclagem de entulho, pois estas além de concederem a destinação final para os resíduos, também são um ponto para a reinserção destes na cadeia produtiva da construção (Paschoalin, Frasson, Camelo, Melo Conti \& Cortese, 2021). Existem muitas pesquisas publicadas referentes à reciclagem de resíduos da construção civil, porém muitas não se preocupam em abordar questões como: gestão, sustentabilidade ou viabilidade econômico-financeira; sendo estes termos importantes para as pesquisas (Gularte, Bortoluzzi, Lima, Pinto \& Santos Goffi, 2018).

Com o aumento da industrialização, das construções urbanas e da concentração de pessoas em espaços territoriais pequenos, a disposição dos resíduos sólidos urbanos se tornou um 
problema para a sociedade (Ferreira \& Tambourgi, 2009). Estes resíduos podem gerar problemas sérios se não forem descartados adequadamente, virando uma preocupação de cada município, o controle da sua destinação correta (Oliveira \& Paschoalin, 2016). Os autores ainda comentam que é possível perceber problemas relacionados com a falta da gestão de resíduos nas comunidades e bairros, onde os RSU são descartados em lugares errados, facilitando a transmissão de doenças e também podendo ocorrer a degradação do local de descarte; tornando assim, para o município, um alto custo para a remoção e descarte correto dos resíduos.

Uma solução para a gestão dos RSU envolve aspectos políticos, geográficos, planejamento local e regional; por isso, a gestão ambiental torna-se muito importante para minimizar os impactos ao meio ambiente (Ferreira \& Tambourgi, 2009). Para que a população ajude na destinação correta dos RSU, é necessário que haja pontos de entrega voluntária em locais acessíveis e próximos dos usuários (Oliveira \& Paschoalin, 2016), mas este problema ainda persiste nos municípios brasileiros.

Neste contexto, entender como ocorre a evolução dos conceitos relacionados a ponto de entrega voluntária e suas relações com a reciclagem, podem promover indicativos para a melhoria na gestão de resíduos sólidos urbanos. Percebe-se também que a identificação de possíveis indicadores e desafios existentes poderá promover uma gestão de resíduos sólidos urbanos adequada ao contexto brasileiro. Assim, o objetivo desta pesquisa é realizar um mapeamento científico sobre a evólução do conceito ponto de entrega voluntária e suas relações com a melhoria no processo de reciclagem dos resíduos sólidos urbanos. Procurou-se avaliar também como a Política Nacional de Resíduos Sólidos contribuiu para a disseminação das Cooperativas de Materiais Recicláveis e como a gestão municipal pode, a partir dos Pontos de Entrega Voluntária (PEV), utilizá-los como instrumentos para o fortalecimento das práticas da coleta seletiva no Brasil.

\section{Referencial teórico}

\subsection{Lei 12.305/2010 (PNRS) e a contribuição para a disseminação das cooperativas de}

\section{materiais recicláveis}

De acordo com a lei 12.305/2010, a coleta seletiva é definida como a "coleta de resíduos sólidos previamente segregados conforme sua constituição ou composição". Tendo também como um de seus instrumentos o incentivo em relação à criação, ao desenvolvimento de cooperativas e de diferentes formas de associação com catadores de resíduos recicláveis e também reutilizáveis. 
Segundo essa lei, a elaboração de planos municipais visando uma gestão integrada dos resíduos sólidos, é uma condição para os municípios terem acesso a recursos da união, visando empreendimentos e serviços relacionados à limpeza urbana e ao manejo dos resíduos sólidos. Os municípios, através destes planos, podem se beneficiarem por meio de incentivos ou financiamentos de recursos federais, e assim, acessarem créditos ou fomentos com esta finalidade - gerir os resíduos sólidos municipais. Em seu artigo § 1o destaca-se que serão priorizados no acesso aos recursos da União, os municípios que implantarem a coleta seletiva com a participação de cooperativas ou outras formas de associação de catadores de materiais reutilizáveis e recicláveis, formadas por pessoas físicas de baixa renda.

O plano municipal de gestão integrada de resíduos sólidos deve apresentar o seguinte conteúdo: programas e ações para a participação dos grupos interessados, em especial das cooperativas ou outras formas de associação de catadores de materiais reutilizáveis e recicláveis, formadas por pessoas físicas de baixa renda, se houver. Neste plano serão estabelecidos por regulamento as normas sobre a exigibilidade e o conteúdo do plano de gerenciamento de resíduos sólidos relativo à atuação de cooperativas ou de outras formas de associação de catadores de materiais reutilizáveis e recicláveis.

Como forma de incentivo ao desenvolvimento das cooperativas de materiais reutilizáveis e recicláveis, o poder público poderá instituir medidas indutoras e linhas de financiamento para atender, prioritariamente, às iniciativas de: implantação de infraestrutura física e aquisição de equipamentos para cooperativas oú outras formas de associação de catadores de materiais reutilizáveis e recicláveis formadas por pessoas físicas de baixa renda. De acordo com Soares et al. (2020), as questões essenciais para que a PNRS se desenvolvesse foram: a logística reversa e também a posição estratégica das cooperativas e dos catadores. Ainda segundo os autores, os catadores são uma parte muito frágil da cadeia, pois eles dependem das indústrias e de atravessadores, por causa dos preços, do volume e também das condições que os materiais são recolhidos.

Na figura 2 é apresentado o gráfico com a compilação dos dados de uma gravimetria nacional. Para a realização da gravimetria, foi feita uma estimativa com a média ponderada da geração total de RSU por faixa de renda dos municípios e das suas gravimetrias, levando em consideração a população e geração per capita (ABRELPE, 2020). 


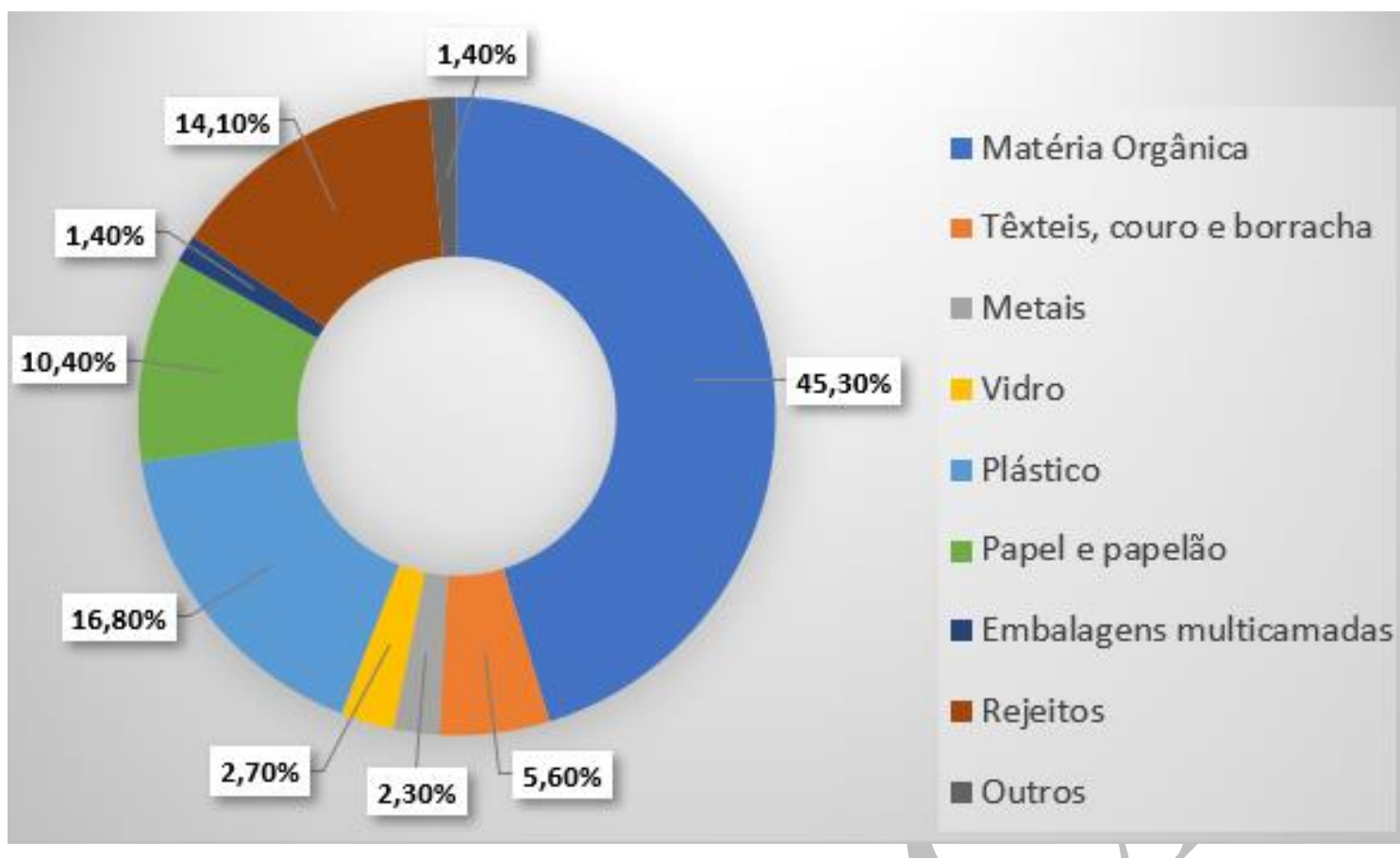

Figura 2 - Gravimetria nacional dos resíduos sólidos urbanos

Fonte: Adaptado de ABRELPE (2020).

Com estas informações, pode-se verificar que a matéria orgânica é o principal componente dos resíduos sólidos urbanos, com 45,3\%. Os resíduos plásticos são o segundo maior grupo, com $16,8 \%$, seguidos de papel e papelão, com 10,4\%; resíduos têxteis, couros e borrachas, com $5,6 \%$; vidros, com $2,7 \%$; metais, com $2,3 \%$; embalagens multicamadas, com $1,4 \%$; outros resíduos, com 1,4\%. Os rejeitos correspondem a 14,1\% do total de resíduos. Conforme estudos do Panorama dos Resíduos Sólidos no Brasil a geração de resíduos está aumentando e estima-se que até 2030, a geração anual de resíduos sólidos no Brasil, será de 100 milhões de toneladas (ABRELPE, 2019).

\subsection{Reciclagem}

A reciclagem é extremamente importante nos dias atuais, pois somente no Brasil, é estimada uma produção de resíduos em torno de 379,2 kg per capita por ano (ABRELPE, 2020). A reciclagem de uma parte destes resíduos pode beneficiar muito o meio ambiente, reduzindo a poluição do ar, da água e também aumentar a vida útil dos aterros sanitários (Nolan, 2017). Para que a reciclagem seja organizada e bem sucedida, os municípios precisam se comprometer com o controle do programa de reciclagem, podendo levar em consideração algumas perspectivas, como: “(i) projetar o programa para atingir uma meta específica de desvio de resíduos; ou (ii) otimizar o projeto considerando compensações entre taxas de desvio mais altas e custos mais altos” (Noehammer \& Byer, 1997). 
Muitas vezes, nos centros de coleta, é dado um incentivo em dinheiro em troca dos materiais reciclados, porém para algumas pessoas, isso não é suficiente para motivar a preparação, o armazenamento e o transporte dos materiais até o local de entrega (Vining, Linn \& Burdge, 1992). Por esse motivo, Noehammer e Byer (1997) realizaram um estudo comparando programas de reciclagem voluntários e obrigatórios, verificando que os programas obrigatórios possuem índices mais altos de participação, mas somente quando há políticas de fiscalização formalizadas. Saphores, Ogunseitan e Shapiro, (2012) identificaram que havendo locais de entrega fixos, é mais fácil implementar programas de reciclagem do que os programas de devolução que envolvem fabricantes, por exemplo.

Nos Estados Unidos, Park e Berry (2013) verificaram que cada condado pode escolher os programas de reciclagem que querem implantar, porém os mais utilizados são: curbside pickup (coleta na calçada), pay-as-you-throw (pague conforme você joga fora), RecycleBank (banco de reciclagem) e recycled product markets (mercados de produtos reciclados). Estes programas de reciclagem podem ser utilizados para residências, indústrias, comércios e instituições de comunidades. Uma consideração importante é que cada setor gera resíduos distintos, então tudo isso precisa ser levado em consideração na hora da coleta e separação dos resíduos (Noehammer \& Byer, 1997).

Lawrence, Cooper e Kissoon (2020) mencionam que muitas pessoas acabam se envolvendo com a reciclagem pelo "amor e preocupação com o meio ambiente", se comprometendo e demonstrando resultados ótimos de reciclagem. Na Suíça, por exemplo, existe a reciclagem de garrafas PET, sendo voluntária e sem sistema de depósito; são coletados resíduos em espaços públicos, comércios e também em centros de coleta municipais, tendo nos últimos anos, um aumento de 60\% nas coletas (Haupt, Waser, Würmli \& Hellweg, 2018).

\subsection{Ponto de Entrega Voluntária}

Os pontos de entrega voluntária são lugares onde os cidadãos podem deixar seus materiais recicláveis para que estes possam, posteriormente, ser encaminhados para reciclagem. Em São Paulo - SP, houve a instalação de um ponto de entrega voluntária para embalagens de poliestireno expandido, os materiais recebidos são comercializados e também transformados em outros produtos (Kerber, Souza, Hassemer \& Bouzon, 2020).

Os centros de coleta seletiva são lugares onde há coleta centralizada de resíduos sólidos municipais (Feo \& Polito, 2015). Foi observado por Islam, Dias e Huda (2020) que muitas pessoas guardam seus lixos eletrônicos em casa até conseguir levá-los para os centros de coleta, sendo esta, a prática mais mencionada entre os resultados da pesquisa. Já um banco de resíduos ensina as pessoas sobre a separação dos resíduos sólidos (Ridley-Duff \& Bull, 2019), 
sendo também um local de armazenamento dos resíduos que podem ser reciclados, ajudando os cidadãos a aumentar sua consciência ambiental (Singhirunnusorn, Donlakorn \& Kaewhanin., 2012; Solihin, Muljono \& Sadono, 2018).

A modalidade de reciclagem drop-off é um programa onde há vários locais para a entrega de materiais recicláveis, onde os recicladores, normalmente, precisam colocar os materiais separados nos recipientes marcados (Sidique, Lupi \& Joshi, 2013). De acordo com Vining et al. (1992), verifica-se que a coleta de resíduos na calçada envolve um programa voluntário de reciclagem "de jornais, vidros, latas e plástico"

\subsection{Indicadores para a gestão dos resíduos sólidos urbanos}

Indicadores podem ser definidos como "um parâmetro ou um valor derivado de parâmetros, que fornece informações sobre um fenômeno", eles também são considerados sintéticos e são feitos para um propósito em específico (Organisation for Economic Co-Operation and Development - OECD, 1993). De acordo com Santiago e Dias (2012), é difícil escolher o indicador ideal, pois, muitas vezes, os assuntos abordados nas pesquisas são muito complexos, necessitando de vários indicadores diferentes, que se complementam. Para a área de gestão de resíduos sólidos, é preciso desenvolver e implementar indicadores, para conseguir avaliar os avanços ou até mesmo o retrocesso dos projetos, visando sempre alcançar as metas estabelecidas da PNRS (Campani, 2012).

Para ser possível entender melhor e alcançar os objetivos propostos nas pesquisas, os indicadores precisam ser compreensíveis e também necessitam de resultados que não sejam apenas numéricos, para que a tomada de decisão possa ser mais precisa (Campani, 2012). Existem, no Brasil, bases de dados oficiais que possuem indicadores de coleta seletiva, como as do IBGE e do Ministério das Cidades. O Sistema Nacional de Informações sobre Gestão de Resíduos Sólidos (SINIR), atende a PNRS possuindo estatísticas e também indicadores sobre serviços públicos e privados de gestão de resíduos (Fechine \& Moraes, 2014).

Existem indicadores de várias áreas diferentes, mas falando somente sobre a sustentabilidade, para Fechine e Moraes (2014), estes indicadores "podem ser entendidos como lentes capazes de enxergar os pontos positivos, pontos frágeis e problemas que acabam inviabilizando o programa de coleta seletiva”. Os autores também dizem que são ferramentas de apoio para o planejamento, gerenciamento e monitorização da coleta seletiva. Os indicadores de sustentabilidade que são projetados em países industrializados, focam mais na parte ambiental dos resíduos (Besen, 2011).

Com relação aos indicadores de gestão de resíduos sólidos e sustentabilidade, foi possível identificar alguns que podem ser utilizados: no controle e monitoramento de resíduos, na 
coleta seletiva, na reciclagem dos resíduos, no monitoramento e eficiência da coleta; indicadores econômicos, incluindo custos e despesas, da coleta e do descarte dos resíduos. Estes indicadores são qualitativos e quantitativos, visando sempre obter uma informação precisa para a análise dos dados coletados. Os indicadores encontrados estão descritos no Quadro 1.

\begin{tabular}{|c|c|}
\hline Autores & Indicadores \\
\hline \multirow{19}{*}{$\begin{array}{l}\text { SISTEMA NACIONAL } \\
\text { DE INFORMAÇÕES } \\
\text { SOBRE SANEAMENTO } \\
\text { (SNIS) (Brasil, 2011) }\end{array}$} & Massa recuperada per capita (kg/hab.dia) \\
\hline & Relação entre quantidades da coleta seletiva e Resíduos Domiciliares (RDO) (\%) \\
\hline & Massa per capita recolhida via coleta seletiva $(\mathrm{kg} / \mathrm{hab} . \mathrm{ano})$ \\
\hline & $\begin{array}{c}\text { Taxa de recuperação de recicláveis em relação à quantidade de Resíduos } \\
\text { Domiciliares (RDO) e Resíduos Públicos (RPU) (\%) }\end{array}$ \\
\hline & Incidência de despesas com Resíduos Sólidos Urbanos (RSU) na prefeitura (\%) \\
\hline & Incidência de despesas com empresas contratadas $(\%)$ \\
\hline & Despesas per capita com RSU (R\$/habitante) \\
\hline & $\begin{array}{c}\text { Taxa de cobertura de coleta direta dos Resíduos Domiciliares (RDO) relativo à } \\
\text { população urbana }(\%)\end{array}$ \\
\hline & $\begin{array}{l}\text { Massa [RDO+RPU] coletada per capita em relação à população urbana }(\mathrm{Kg} /(\text { hab.x } \\
\text { dia) })\end{array}$ \\
\hline & Custo unitário da coleta $(\mathrm{R} \$ /$ tonelada $)$ \\
\hline & Incidência do custo da coleta no custo total do manejo (\%) \\
\hline & Taxa de cobertura da coleta seletiva porta-a-porta em relação a população urbana (\%) \\
\hline & Taxa de recuperação de recicláveis em relação à quantidade de RDO e RPU (\%) \\
\hline & Relação entre quantidades da coleta seletiva e RDO $(\%)$ \\
\hline & Incidência de papel/papelão sobre o total de material recuperado (\%) \\
\hline & Incidência de plásticos sobre o total de material recuperado (\%) \\
\hline & Incidência de metais sobre o total de material recuperado (\%) \\
\hline & Incidência de vidros sobre o total de material recuperado (\%) \\
\hline & Incidência de "outros" sobre o total de material recuperado (\%) \\
\hline \multirow{2}{*}{ PNSB (IBGE, 2010) } & Área de abrangência do serviço de coleta seletiva \\
\hline & Existência de cooperativas ou associações no município \\
\hline Besen (2011) & Instrumentos legais na relação com organização de catadores \\
\hline Ribeiro et al. (2009) & Parcerias entre as organizações \\
\hline $\begin{array}{l}\text { Bringhenti (2004); Lima } \\
\text { (2006); Milanez (2002); } \\
\text { Besen (2011); Campani } \\
\text { (2012) }\end{array}$ & Custo total do programa em relação a quantidade coletada seletivamente ( $R \$ / t)$ \\
\hline Fechine e Moraes (2014) & $\begin{array}{c}\text { Relação entre despesas com coleta seletiva e despesas empregadas com limpeza } \\
\text { urbana }(\%)\end{array}$ \\
\hline $\begin{array}{l}\text { Lima (2006); Besen } \\
\text { (2011); Duarte (2012) }\end{array}$ & Taxa de rejeitos $(\%)$ \\
\hline Lima (2006) & $\begin{array}{c}\text { Taxa de material recolhido nos Pontos de Entrega Voluntária (PEVs) em } \\
\text { relação a quantidade total coletada pela coleta seletiva (\%) }\end{array}$ \\
\hline Campani (2012) & Reciclabilidade \\
\hline \multirow{4}{*}{$\begin{array}{l}\text { Instituto de pesquisa } \\
\text { econômica aplicada - } \\
\text { IPEA (2010) }\end{array}$} & Número de catadores cooperados \\
\hline & Produtividade física por cooperativa \\
\hline & Produtividade física média por catador \\
\hline & Peso de materiais vendidos por tipo de material \\
\hline \multirow{5}{*}{ Santiago e Dias (2012) } & Eficiência de coleta \\
\hline & Existência de lixeiras públicas \\
\hline & Existência de coleta seletiva no município \\
\hline & Abrangência da coleta seletiva no município \\
\hline & Existência de PEVs dos resíduos segregados \\
\hline
\end{tabular}




\begin{tabular}{|c|c|}
\hline \multirow{4}{*}{} & Índice de recuperação de materiais recicláveis \\
\cline { 2 - 2 } & Geração de resíduos sólidos urbanos per capta \\
\cline { 2 - 2 } & Aterro sanitário controlado/licenciado \\
\cline { 2 - 2 } & Existência de aterro para resíduos de construção e demolição \\
\hline
\end{tabular}

Quadro 1 - Indicadores de gestão de resíduos sólidos

Fonte: Autores (2021).

Como sugestão para que os pontos de entrega voluntária sejam instrumentos de gestão dos municípios, pode-se adotar os 16 indicadores assinalados em negrito dentro do quadro 1 , assim a gestão municipal poderá, a partir das informações geradas pelos indicadores, pensar em estruturar pontos de entrega voluntária em outros bairros nos municípios, utilizando-se da PNRS para vislumbrar acessos a recursos da União e tratar de forma mais profissionalizada a limpeza urbana e o manejo dos resíduos sólidos urbanos.

\section{Metodologia}

O primeiro passo de qualquer pesquisador ao desenvolver um trabalho é construir conhecimento em determinado contexto, e isto ocorre por meio da revisão da literatura sobre o tema (AFONSO et al., 2011). A revisão de literatura busca informar ao leitor sobre o que foi aprendido, destacando os padrões de convergências e divergências encontrados na literatura e as perspectivas para futuros temas emergentes (Webster \& Watson, 2002), e pode ocorrer de diversas formas. Uma delas é a análise bibliométrica. Segundo Araújo (2006), para a realização de estudos bibliométricos, realiza-se uma leitura de dados bibliométricos à luz de elementos do contexto sócio-histórico em que a atividade científica é produzida. Para iniciar a análise bibliométrica foram construídas questões de pesquisa que respondam ao objetivo deste estudo. Para responder às questões de pesquisas, foi realizada uma análise bibliométrica, e as etapas metodológicas utilizadas são descritas no fluxograma da figura 3. 


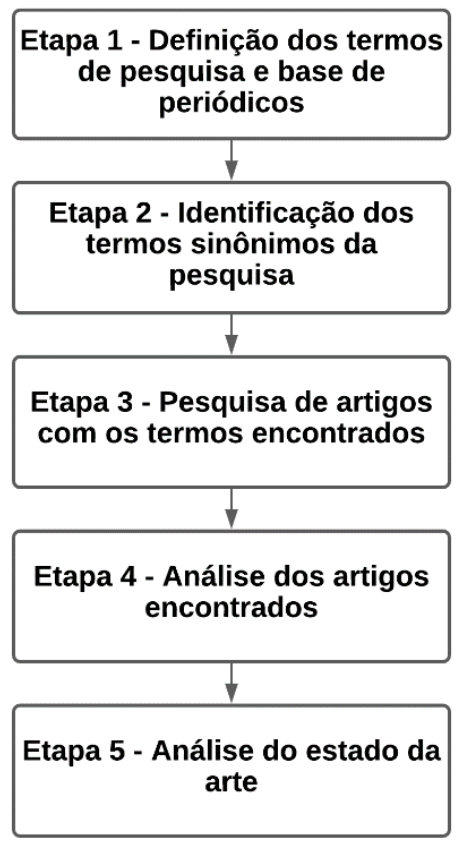

Figura 3 - Etapas metodológicas

Fonte: Autores (2021).

Etapa 1 - Definição dos termos de pesquisa e base de periódicos: primeiramente foi definida a base de dados Scopus. Selecionou-se esta base de pesquisa, pois segundo Oliveira e Gracio (2011), ela é definida como "uma base de dados multidisciplinar, lançada pela editora Elsevier, em 2004”. Ela também possui qualidade de dados, análises qualificadas e tecnologias avançadas, o que transmite aos investigadores, que se está pesquisando em uma base de dados confiável (Scopus, 2021).

Na primeira rodada da pesquisa bibliográfica, foram definidos dois termos de pesquisa: "locais de entrega voluntária" e "ponto de entrega voluntária". Os termos foram pesquisados, em inglês "voluntary delivery point" e "voluntary delivery locations", na base de dados Scopus. O termo "voluntary delivery locations", não teve nenhum resultado encontrado e o termo "voluntary delivery point" apresentou 4 resultados.

Etapa 2 - Identificação dos termos sinônimos da pesquisa: os 4 artigos encontrados foram lidos e, com eles, foi possível encontrar palavras sinônimas para os termos de pesquisa; também foi realizada uma pesquisa com os termos separados: "voluntary” AND "location" AND "recycling"; "voluntary" AND "delivery" AND "recycling”. Os termos encontrados foram: "disposal points", "voluntary recycling programs", "voluntary recycling projects", "recycling programs".

Etapa 3 - Pesquisa de artigos com os termos encontrados: os termos destacados da etapa 2 foram pesquisados separadamente, juntamente com a palavra "recycling", para que fossem encontrados resultados específicos sobre reciclagem. Novas palavras semelhantes foram 
encontradas: "drop-off locations", "drop-off centers", “curbside pickup”, "recyclable pickup”, "collection points", "collection centers", "waste bank".

Etapa 4 - Análise dos artigos encontrados: depois dos termos serem separados, foi realizada uma pesquisa com cada um dos termos, selecionando artigos ou revisões, que tratassem sobre reciclagem e fossem interessantes para esta pesquisa, a partir de 1992, pois foi depois deste ano que as publicações aumentaram. Estes artigos foram analisados, identificando o que cada um apresentava sobre o sinônimo abordado e também sobre sugestões para futuros trabalhos. Após isso, foi utilizada a string "("recycling”) AND ("voluntary recycling program*” OR "voluntary recycling project*” OR "recycling program*” OR "drop-off location*” OR "dropoff center*” OR “disposal point*” OR "voluntary delivery point*” OR "curbside pickup*” OR "recyclable pickup*" OR "collection point*" OR "collection center*" OR "waste bank*")" para realizar uma pesquisa mais abrangente. Foram selecionados artigos a partir de 1992; somente revisões e artigos foram selecionados; e por último, somente pesquisas realizadas na língua inglesa. Com esta pesquisa foram encontrados 4095 resultados. Estes foram analisados com o auxílio do software SciMAT, avaliando a evolução temática e o estado da arte sobre pontos de entrega voluntária e sua relação com a reciclagem; os artigos foram divididos em 6 períodos de 5 anos cada um. O SciMAT foi proposto por Cobo, LópezHerrera, Herrera-Viedma e Herrera (2012), sendo este um instrumento utilizado (um software) para mapeamento científico. Com um conjunto de documentos, ele gera uma base de conhecimento, podendo conter autores, palavras-chave, periódicos e referências, ajudando o pesquisador a realizar o mapeamento científico, desde o carregamento dos dados até a interpretação dos resultados (Cobo et al., 2012).

Etapa 5 - Análise do estado da arte: com o uso do SciMAT foram selecionados artigos de cada termo sinônimo de pesquisa. Estes artigos foram lidos na íntegra e serviram de subsídio para a interpretação e discussão dos pesquisadores. O objetivo da leitura foi verificar como cada autor define o termo relacionado a pontos de entrega voluntária e quais suas contribuições para a melhoria do processo de reciclagem e a sua participação como instrumento de gestão municipal dos resíduos sólidos urbanos.

Para a análise dos termos encontrados foi utilizado o software SciMAT. Diagramas dos temas motores por período foram desenvolvidos para análise da evolução e relações entre pontos de entrega voluntária e a reciclagem.

\section{Resultados e discussão}

Realizando a pesquisa sobre os termos selecionados, em artigos e/ou revisões, foi possível verificar que, na base de dados Scopus, o primeiro termo a ser utilizado, foi "disposal point" 
em 1913. Depois surgiram os termos "collection center", “collection point”, "recycling program”, “waste bank”, “drop-off center”, "voluntary recycling program”, “drop-off location”, “curbside pickup”, “recyclable pickup”, “voluntary delivery point” nesta ordem (Figura 4). 


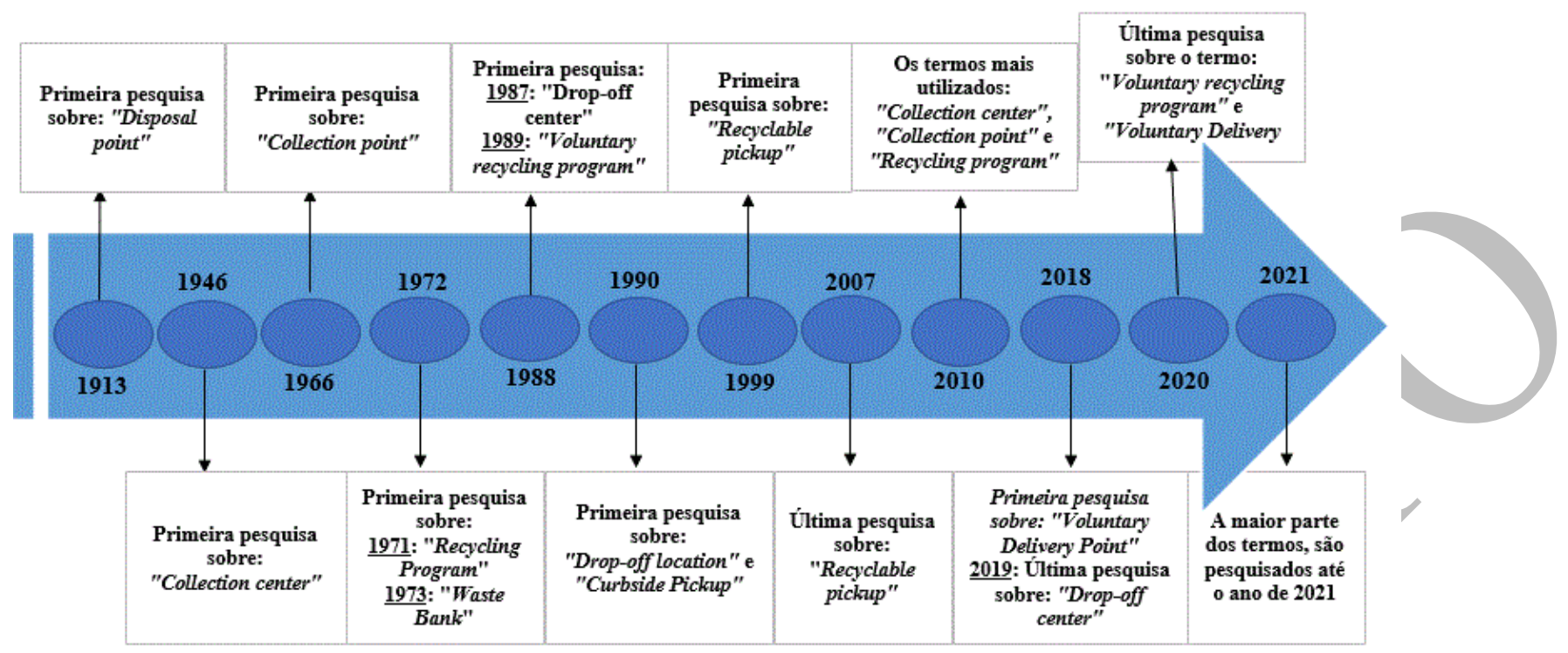

Figura 4 - Linha do tempo dos termos de pesquisa

Fonte: Autores (2021).

No início do século XX, mais precisamente em 1913, surgiu o primeiro termo "ponto de descarte", pesquisado pelo autor Wingate T. Todd, sendo um termo pesquisado até os dias atuais e tendo como principais autores durante os anos: Anon; Delvigne, G.A.L.; Lafayette, K.P.V.; Pitman, J.S.; Riera, R. Já em 1946 houve a primeira pesquisa com o termo "centro de coleta", pelo autor Paul Hauduroy, sendo também pesquisado até hoje. Este termo é o primeiro que remete ao conceito de coleta de resíduos sólidos, tendo como autores principais: Tavakkoli-Moghaddam, R.; Basavaraju, S.V. A partir de 1970, os termos começam a aumentar, assim como os resultados de pesquisas, surgindo termos como "banco de resíduos", "programa de reciclagem", "programa de reciclagem voluntária", "centro de entrega", "local de entrega", "coleta na calçada" e "coleta reciclável”. Havendo também em 1972 a Conferência de Estocolmo, onde foram decididas questões ambientais internacionais, como: gestão dos resíduos naturais, direitos humanos, prevenção da poluição, entre outras (Organização das Nações UnidasONU, 2017); o que contribuiu para a disseminação de estudos e publicações. Em 2010, foi possível verificar que os termos mais pesquisados foram "centro de coleta", "ponto de coleta" e "programa de reciclagem". Comparando os termos em 2010 com a sua definição que foi primeiramente definida é possível perceber que elas não mudaram no conceito geral, somente foram aperfeiçoadas. Centros de coleta são abordados como locais de disposição de resíduos, mas a diferença é que em 1996, eram, na maioria, catadores que realizavam a entrega de materiais (Kaseva \& Gupta, 1996), já em 2015, a população está mais envolvida na entrega e vai até os centros deixar seus 


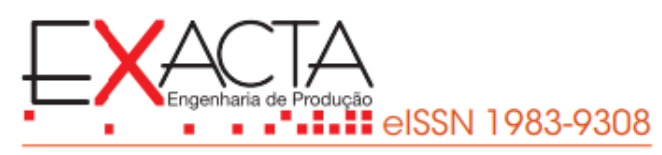

materiais recicláveis. O termo ponto de coleta é o mesmo durante os anos, corresponde a um local de coleta de resíduos sólidos urbanos (Lee, Chang, Wang \& Wen, 2000;

Alvarez, Larrucea, Quero \& Valle, 2008; Haupt et al., 2018; Islam et al., 2020). Programas de reciclagem possuíam, em 1997, alguns problemas, como custo de implementação altos; a população não participava ativamente; mercados que variam em relação aos materiais recicláveis, entre outros (Noehammer \& Byer, 1997); já em 2016, nos Estados Unidos, é comentado em vários programas de reciclagem, como coleta na calçada, pague conforme você joga fora, banco de reciclagem e mercados de produtos reciclados (Park \& Berry, 2013)

Recentemente, em 2018, o termo ponto de entrega voluntária surgiu, possuindo apenas 4 resultados de pesquisa. Com isso, percebe-se que o termo é recente e que ainda existem poucos resultados encontrados sobre ele. A última pesquisa do termo foi em 2020, sendo os autores dos quatro resultados: Ferreira, César, Conejero e Guabiroba (2020); Klein, Gonçalves-Dias e Jayo (2020); Paz, Lafayette e Sobral (2020); Kerber, Antunes de Souza Júnior, Hassemer e Bouzona (2020).

Foi utilizado o software SciMAT para analisar os temas motores sobre pontos de entrega voluntária e sua relação com a reciclagem, bem como para identificar boas práticas. O mapeamento mostrou que na utilização da palavra "Municipality" (Município), no período de 2002 a 2006, obteve-se a palavra "scavenger" (Catador); o termo "3R", teve como resultado "waste bank" (banco de resíduo), no período de 2012 a 2016. No período de 2017 a 2021, surgiram os termos: "Social Movement" (Movimento Social), que se relacionou com a palavra "cooperatives" (cooperativas); já o termo "Solid Waste" (Resíduo Sólido), que também apareceu no mesmo período (de 2017 a 2021), apresentou uma forte relação com a palavra "waste bank" (banco de resíduo); outro termo que também apareceu no mesmo período foi "Municipal Solid Waste" (Resíduo Sólido Municipal), o qual se relaciona fortemente com as palavras "scavenger" (catador), "indicators" (indicadores) e "recyclables" (recicláveis); outro termo que também foi percebido na pesquisa no mesmo período foi "Reverse Logistics" (Logística Reversa), a qual apresentou uma ligação bastante intensa com as palavras "collection points" (pontos de coleta) e "waste" (resíduo).

Com o passar dos anos, o número de artigos publicados aumentou e consequentemente o número de termos citados em cada um, com isso, é visível, que com o passar dos anos, os termos possuem maiores ligações com outros termos. Principalmente nos últimos 10 anos, as ligações aumentaram mais ainda. Também é possível perceber que os PEVs 
possuem ligações diretas com a reciclagem, pois os materiais recicláveis precisam ser deixados nestes locais de coleta para poderem seguir um fluxo de Economia circular que faça com que os mesmos sejam novamente reaproveitados nos ciclos produtivos. A população, com isso, precisa saber como separar os materiais e como entregá-los corretamente. É necessário haver uma conscientização da população para o descarte correto dos materiais nos pontos de entrega voluntária.

Em relação aos indicadores encontrados segundo os autores: Brasil, 2011; Bringhenti, 2004; Lima, 2006; Milanez, 2002; Besen, 2011; Campani, 2012; Duarte, 2012; IPEA, 2010; Santiago \& Dias, 2012, selecionou-se dentre eles 16, que poderão ser utilizados visando qualificar a gestão municipal dos Resíduos Sólidos Urbanos; sendo eles apresentados no Quadro 2:

\begin{tabular}{|c|c|}
\hline Indicador & Autores \\
\hline Massa recuperada per capita (kg/hab.dia) & \multirow{10}{*}{$\begin{array}{c}\text { SISTEMA NACIONAL DE } \\
\text { INFORMAÇÕES SOBRE SANEAMENTO } \\
\text { (SNIS) (Brasil, 2011) }\end{array}$} \\
\hline $\begin{array}{l}\text { Relação entre quantidades da coleta seletiva e Resíduos } \\
\text { Domiciliares (RDO) }(\%)\end{array}$ & \\
\hline $\begin{array}{l}\text { Taxa de recuperação de recicláveis em relação à } \\
\text { quantidade de Resíduos Domiciliares (RDO) e } \\
\text { Resíduos Públicos (RPU) (\%) }\end{array}$ & \\
\hline $\begin{array}{l}\text { Taxa de cobertura de coleta direta dos RDO relativo à } \\
\text { população urbana }(\%)\end{array}$ & \\
\hline $\begin{array}{l}\text { Taxa de recuperação de recicláveis em relação à } \\
\text { quantidade de RDO e RPU }(\%)\end{array}$ & \\
\hline $\begin{array}{l}\text { Incidência de papel/papelão sobre o total de material } \\
\text { recuperado }(\%)\end{array}$ & \\
\hline $\begin{array}{l}\text { Incidência de plásticos sobre o total de material } \\
\text { recuperado }(\%)\end{array}$ & \\
\hline $\begin{array}{l}\text { Incidência de metais sobre o total de material } \\
\text { recuperado }(\%)\end{array}$ & \\
\hline $\begin{array}{l}\text { Incidência de vidros sobre o total de material } \\
\text { recuperado }(\%)\end{array}$ & \\
\hline $\begin{array}{l}\text { Incidência de "outros" sobre o total de material } \\
\text { recuperado }(\%)\end{array}$ & \\
\hline $\begin{array}{l}\text { Custo total do programa em relação a quantidade } \\
\text { coletada seletivamente }(\mathrm{R} \$ / \mathrm{t})\end{array}$ & $\begin{array}{l}\text { Bringhenti (2004); Lima (2006); Milanez } \\
\text { (2002); Besen (2011); Campani (2012) }\end{array}$ \\
\hline Taxa de rejeitos $(\%)$ & Lima (2006); Besen (2011); Duarte (2012) \\
\hline $\begin{array}{l}\text { Taxa de material recolhido nos Pontos de Entrega } \\
\text { Voluntária (PEVs) em relação a quantidade total } \\
\text { coletada pela coleta seletiva (\%) }\end{array}$ & Lima (2006) \\
\hline Peso de materiais vendidos por tipo de material & $\begin{array}{c}\text { Instituto de pesquisa econômica aplicada - } \\
\text { IPEA (2010) }\end{array}$ \\
\hline $\begin{array}{l}\text { Existência de PEVs dos resíduos segregados } \\
\text { Índice de recuperação de materiais recicláveis }\end{array}$ & Santiago e Dias (2012) \\
\hline
\end{tabular}

Quadro 2 - Indicadores selecionados

Fonte: Autores (2021).

Estes indicadores abordam tanto as taxas de recolhimento de materiais recicláveis, como papel, papelão, plástico, metal, vidro, entre outros, como também custo de 
implementação de programas de coleta seletiva e a existência de pontos de entrega voluntária em determinado lugar.

Os pontos de entrega voluntários possuem algumas inconveniências que precisam ser levados em consideração. Vining et al. (1997) afirmam que existe uma pressão social muito grande por parte de vizinhos e/ou familiares em relação a reciclagem, o que faz com que as pessoas não reciclam, mesmo que os cidadãos estejam motivados e acreditem que a reciclagem traz bons resultados para o meio ambiente, o tempo, espaço e trabalho para realizar a preparação, armazenamento e transporte dos materiais desmotivam muita gente. A idade é um fator que influencia muito na reciclagem, jovens e idosos tendem a não participar tanto de programas de reciclagem; gênero também é um fator influenciador, mulheres tendem a estar mais engajadas em projetos para ajudar o meio ambiente (Saphores, Nixon, Ogunseitan \& Shapiro, 2006). Para Sidique et al. (2013), se as pessoas não estiverem familiarizadas e não tiverem uma certa conveniência, elas não estarão tão dispostas a reciclagem.

Alguns autores, no final de suas pesquisas, indicaram sugestões para trabalhos futuros (Quadro 3).

\begin{tabular}{|c|c|}
\hline Sugestões para trabalhos futuros & Autores \\
\hline $\begin{array}{c}\text { Mais pesquisas são necessárias a fim de verificar a relação existente entre o } \\
\text { método de coleta e a taxa de participação da população; para estabelecer o } \\
\text { impacto da frequência da coleta na taxa de participação; e também estabelecer } \\
\text { qual seria a relação entre os incentivos econômicos e a taxa de participação. }\end{array}$ & $\begin{array}{c}\text { Noehammer e } \\
\text { Byer (1997) }\end{array}$ \\
\hline $\begin{array}{c}\text { Sugere-se investigações adicionais em opções de reciclagem e também uma } \\
\text { tentativa de ter uma compreensão da disposição de reciclar em grupos } \\
\text { minoritários. }\end{array}$ & $\begin{array}{c}\text { Saphores } \text { et al. } \\
(2006)\end{array}$ \\
\hline $\begin{array}{c}\text { Indicam explorar melhor quais são os obstáculos para a reciclagem de lixo } \\
\text { eletrônico nos grupos minoritários; comparar qual é a eficácia das opções de } \\
\text { reciclagem de lixo eletrônico; e também, avaliar qual seria a viabilidade de } \\
\text { incentivos econômicos. }\end{array}$ & $\begin{array}{c}\text { Saphores } \text { et al. } \\
(2012)\end{array}$ \\
\hline $\begin{array}{c}\text { É preciso avaliar sistemas de gestão de resíduos sólidos levando em consideração } \\
\text { uma perspectiva de sistema e os efeitos não lineares. }\end{array}$ & $\begin{array}{c}\text { Haupt } \text { et al. } \\
(2018)\end{array}$ \\
\hline $\begin{array}{c}\text { É interessante realizar trabalhos futuros verificando incentivos aos consumidores } \\
\text { participantes de programas de reciclagem e razões para as pessoas não } \\
\text { participarem de programas de reciclagem. }\end{array}$ & \begin{tabular}{c} 
Islam et al. (2020) \\
\hline \multirow{2}{*}{ Quadro 3 - Sugestões para trabalhos futuros }
\end{tabular} \\
\hline
\end{tabular}

Fonte: Autores (2021).

\section{Conclusão}

A gestão municipal, fazendo uso dos indicadores sugeridos, poderá estruturar e utilizar dos pontos de entrega voluntária, juntamente com o que preconiza a PNRS, para a melhoria da gestão dos RSU. Essa ação contribuirá para uma melhor qualidade de vida da população que executa os serviços internamente nos PEVs (catadores), além de elevar a educação ambiental de toda a população do município. À medida que mais 


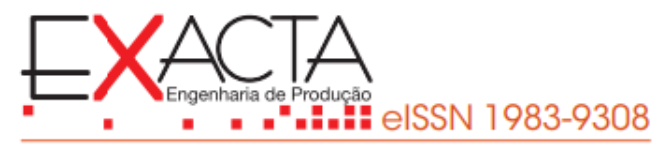

pontos de entrega voluntária forem sendo estruturados dentro do município, outros benefícios ambientais também serão percebidos como: a segregação de um maior volume de materiais recicláveis; notar-se-á uma cidade mais limpa e organizada e consequentemente uma maior qualidade do ar que a população respira.

A gestão municipal tenderá apresentar uma sistemática de gerenciamento dos seus resíduos sólidos municipais, com a participação da coleta seletiva e dos catadores de materiais recicláveis inseridos neste trabalho de segregação e venda dos materiais. O reflexo desta ação será a redução da taxa de limpeza do município, pois haverá menos resíduos gerados e que necessitem ser encaminhados a aterros, havendo menos despesas para a população. A conversão de resíduos segregados em matérias-primas para as empresas, tenderá a se elevar e os ganhos com as vendas destes materiais ficârá de posse dos catadores, fazendo com que os recursos circulem no próprio município. Trata-se de um círculo virtuoso em que todos ganham e o meio ambiente também.

Havendo uma preocupação maior com a gestão dos resíduos sólidos urbanos, mais pesquisas podem ser realizadas, utilizando os indicadores propostos neste trabalho. Como limitação deste estudo ressaltamos que não foram ayaliadas como são as estruturas dos PEVs no Rio Grande do Sul e no Brasil. Como sugestão de trabalhos futuros, sugerimos uma investigação aprofundada sobre como são estruturados os PEVs, para que seja possível aplicar os conceitos na prática. Nos estudos práticos em PEVs sugere-se também a aplicação dos indicadores propostos, verificando a utilidade de cada um.

As questões norteadoras que conduziram esta pesquisa foram respondidas. A primeira questão foi: entender como ocorre a evolução dos conceitos relacionados a ponto de entrega voluntária e suas relações com a reciclagem. A questão foi respondida com a figura 4, onde foi possível identificar os termos mais pesquisados desde 1913. Todos os termos encontrados se relacionam com a reciclagem, pois os resíduos deixados nesses PEVs são, na sua maioria, reciclados. A segunda questão foi: identificar possíveis indicadores e desafios existentes para promover uma gestão de resíduos sólidos urbanos adequada ao contexto brasileiro. Os indicadores selecionados estão dispostos no quadro 2, juntamente com os autores que os mencionam. Os indicadores abordam vários temas, como taxas de recolhimento de materiais recicláveis, implementação de programas de coleta seletiva e a existência de PEVs em determinados lugares. Existem alguns desafios para a gestão de RSU, como por exemplo, custos altos para a implementação 


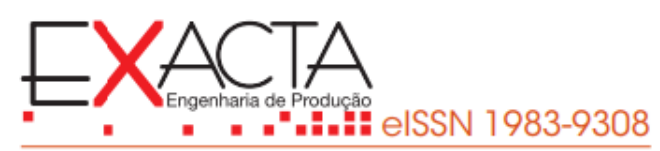

de programas, a não participação da sociedade e a variação dos preços dos materiais recicláveis.

\section{Referências}

Afonso, M. H., Souza, J. D., Ensslin, S. R., \& Ensslin, L. (2011). Como construir conhecimento sobre o tema de pesquisa? Aplicação do processo Proknow-C na busca de literatura sobre avaliação do desenvolvimento sustentável. Revista de Gestão Social e Ambiental, 5(2), 47-62.

Alvarez, J. L., Larrucea, M. A., Quero, S. F. C., \& Valle, A. J. del (2008). Optimizing the collection of used paper from small businesses through GIS techniques: The Leganés case (Madrid, Spain). Waste Management, 28(2), 282-293. Recuperado em 20 junho, 2021, de https://doi.org/10.1016/j.wasman.2007.02.036

Araújo, C. A. (2006). Bibliometria: evolução histórica e questões atuais. Em questão, 12(1), 11-32.

Associação Brasileira das Empresas de Limpeza Pública e Resíduos EspeciaisABRELPE, (2019). Panorama dos Resíduos Sólidos no Brasil 2018/2019. Recuperado em 25 junho, 2021, de https://abrelpe.org.br/panorama/.

Associação Brasileira das Empresas de Limpeza Pública e Resíduos EspeciaisABRELPE, (2020). Panorama dos Resíduos Sólidos no Brasil 2020. Recuperado em 10 maio, 2021, de https://abrelpe.org.br/panorama/.

Batista, T. A., Oliveira, J. A., \& Oliveira, L. D. P. D. S. de (2016). A gestão de resíduos sólidos como instrumento de promoção social. Linguagem Acadêmica, 6(3), 928. Recuperado em 20 junho, 2021, de https://goo.g1/Uj22eA

Besen, G. R. (2011). Coleta seletiva com inclusão de catadores: construção participativa de indicadores e índices de sustentabilidade. Tese de doutorado, Faculdade de Saúde Pública da USP, São Paulo, SP, Brasil. Recuperado em 10 junho, 2021, de https://edisciplinas.usp.br/pluginfile.php/190333/mod_resource/content/1/GinaR izpahBesen.pdf

BRASIL. [Constituição (2010)]. Constituição da República Federativa do Brasil. Brasília, DF: Senado Federal, 2010. 


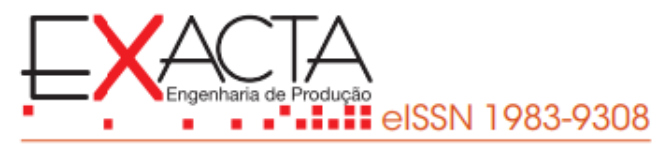

https://www.ipea.gov.br/portal/index.php?option=com_content\&view=article\&i $\mathrm{d}=8858$

Islam, M. T., Dias, P., \& Huda, N. (2020). Waste mobile phones: A survey and analysis of the awareness, consumption and disposal behavior of consumers in Australia. Journal of Environmental Management, 275, 111111. Recuperado em 15 junho, 2021, de https://doi.org/10.1016/j.jenvman.2020.111111

Kaseva, M. E., \& Gupta, S. K. (1996). Recycling — an environmentally friendly and income generating activity towards sustainable solid waste management. Case study_-Dar es Salaam City, Tanzania. Resources, conservation and recycling, 17(4), 299-309. Recuperado em 06 julho, 2021, de https://doi.org/10.1016/S0921-3449(96)01153-6

Kerber, J. C., Souza, H. R. A. D., Hassemer, M. E. N., \& Bouzon, M. (2020). Preliminary study for implementation of voluntary delivery points of expanded polystyrene: a case in southern Brazil. Production, 30. Recuperado em 15 julho, 2021, de https://doi.org/10.1590/0103-6513.20190069

Kipper, L. M., Mahlmann, C. M., \& Rodríguez, A. L. (2009). Ações estratégicas sistêmicas visando à integração da cadeia produtiva e de reciclagem de plásticos. Revista Produção Online, 9(4). Recuperado em 05 junho, 2021, de https://doi.org/10.14488/1676-1901.v9i4.348

Lawrence, K., Cooper, V., \& Kissoon, P. (2020). Sustaining voluntary recycling programmes in a country transitioning to an integrated solid waste management system. Journal of environmental management, 257, 109966. Recuperado em 22 junho, 2021, de https://doi.org/10.1016/j.jenvman.2019.109966

Lee, C. H., Chang, S. L., Wang, K. M., \& Wen, L. C. (2000). Management of scrap computer recycling in Taiwan. Journal of hazardous materials, 73(3), 209-220. Recuperado em 13 junho, 2021, de https://doi.org/10.1016/S03043894(99)00191-0

Lima, R. M. S. R. (2006). Implantação de um programa de coleta seletiva porta a porta com inclusão de catadores: estudo de caso em Londrina - PR. Dissertação de mestrado, Universidade Estadual de Londrina, Londrina, PR, Brasil. Recuperado em 06 junho, 2021, de http://www.uel.br/pos/enges/dissertacoes/29.pdf 


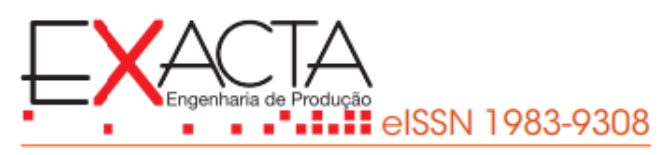

Maiello, A., Britto, A. L. N. P., \& Valle, T. F. (2018). Implementação da Política Nacional de Resíduos Sólidos. Revista de Administração Pública, 52, 24-51. Recuperado em 15 julho, 2021, de https://doi.org/10.1590/0034-7612155117

Milanez, B. (2002). Resíduos sólidos e sustentabilidade. Princípios, indicadores e instrumentos de ação. Dissertação de mestrado, Universidade Federal de São Carlos, São Carlos, SP, Brasil.

Noehammer, H. C., \& Byer, P. H. (1997). Effect of design variables on participation in residential curbside recycling programs. Waste Management \& Research, 15(4), 407-427. Recuperado em 20 junho, 2021, de https://doi.org/10.1006/wmre.1996.0096

Nolan, J. M. (2017). Environmental policies can buttress conservation norms. Society \& Natural Resources, 30(2), 228-244. Recuperado em 22 junho, 2021, de https://doi.org/10.1080/08941920.2016.1209266

Oliveira, E. F. T. D., \& Gracio, M. C. C. (2011). Indicadores bibliométricos em ciência da informação: análise dos pesquisadores mais produtivos no tema estudos métricos na base Scopus. Perspectivas em Ciência da Informação, 16, 16-28. Recuperado em 05 julho, 2021, de https://doi.org/10.1590/S141399362011000400003

Oliveira, R. B. de, \& Paschoalin, J. A., Filho (2016). Mapa interativo para a localização de pontos de entrega voluntária de resíduos recicláveis na cidade de São Paulo. Exacta, 14(2), 163-172. Recuperado em 15 julho, 2021, de https://doi.org/10.5585/exactaep.v14n2.6169

Organização das Nações Unidas-ONU (2017). REPORT OF THE UNITED NATIONS CONFERENCE ON THE HUMAN ENVIRONMENT. Recuperado em 05 junho, 2021, de http://www.un-documents.net/aconf48-14r1.pdf

Organisation for Economic Co-Operation and Development - OECD (1993). OECD core set. Paris: Organization for Economic Co-operation and Development.

Park, S., \& Berry, F. S. (2013). Analyzing effective municipal solid waste recycling programs: the case of county-level MSW recycling performance in Florida, USA. Waste management \& research, 31(9), 896-901. Recuperado em 03 julho, 2021, de https://doi.org/10.1177\%2F0734242X13495725 
https://www.elsevier.com/solutions/scopus?dgcid=RN_AGCM_Sourced_30000 5030

Sidique, S. F., Lupi, F., \& Joshi, S. V. (2013). Estimating the demand for drop-off recycling sites: A random utility travel cost approach. Journal of environmental management, 127, 339-346. Recuperado em 22 julho, 2021, de https://doi.org/10.1016/j.jenvman.2013.05.001

Singhirunnusorn, W., Donlakorn, K., \& Kaewhanin, W. (2012). Contextual factors influencing household recycling behaviours: A case of waste bank project in Mahasarakham Municipality. Procedia - Social and Behavioral Sciences 36, 688-697. Recuperado em 15 julho, 2021, de https://doi.org/10.1016/j.sbspro.2012.03.075

Soares D. G., Barbosa, C. J., Vieira, E. R. \& Silva, A. F. (2020). Cooperativa de catadores e logística reversa: um estudo de caso na cidade de Goiânia, Goiás. Recuperado em 20 junho, 2021, de https://doi.org/10.37423/2020.edc1191

Solihin, M., Muljono, P., \& Sadono, D. (2018). Sustainable waste management through trash bank in Ragajaya Village, Bogor Regency, West Java Province, Indonesia. International Journal of Progressive Sciences and Technologies, 9(2), 190-198. Recuperado em 05 julho, 2021, de http://dx.doi.org/10.52155/ijpsat.v9.2.506

Vining, J., Linn, N., \& Burdge, R. J. (1992). Why recycle? A comparison of recycling motivations in four communities. Environmental management, 16(6), 785-797. Recuperado em 25 junho, 2021, de https://doi.org/10.1007/BF02645669

Webster, J., \& Watson, R. T. (2002). Analyzing the past to prepare for the future: Writing a literature review. MIS quarterly, xiii-xxiii. 